\title{
Sensor less Operation of CSI Fed Induction Motor Drive by Using MRAS
}

\author{
P. Divya Jyothsna ${ }^{1}$, S. Harinath ${ }^{2}$ \\ ${ }^{I}$ (Department of EEE, Govt. Poly technical College, Vempalli, kadapa(Dist), A.P. India) \\ ${ }^{2}$ (Departmentof EEE, PG Scholor, JNTU Anantapur,Anantapuramu (Dist), A.P, India)
}

\begin{abstract}
The underground mines, oil and gas industries need remote operation of vector controlled medium voltage variable speed induction motor drives with long feeder. The usage of voltage source inverters in such cases leads to motor over voltage and harmonic problems. The current source inverter (CSI) has no such problems to these applications because of its motor friendly voltages output. This paper proposes "sensor less operation of a CSI fed induction motor drive" and improves the speed estimation. The proposed model uses model reference adaptive system (MRAS) speed estimator requires motor voltage and currents as inputs. Direct measurement of motor voltages and currents are impractical for long feeder drives due to feeder length and safety of environmental precautions. Hence the motor voltages and currents are drawn from the inverter out. In this paper the flux estimation needed for MRAS speed estimation is proposed to observe the performance of the induction motor with sudden change of loads under steady state. This model is simulated in MATLAB/SIMULINK.
\end{abstract}

Keywords: Induction motor drive, Current source inverter, Model reference adaptive system (MRAS).

\section{Introduction}

Now a day's mostly the voltage source inverter fed induction motors are used in many applications. For medium voltage applications like underground mines, electrical submergible pumps and ventilating pumps with high length of feeder the voltage source inverter fed drives are incurs many problems. The current source inverter fed induction motor drive has many advantages such as short circuit proof operation. Induction motor with 5H.P rating is used from the Matlab/Simulink inbuilt block for the analysis of motor under steady state condition with sudden change of load as $2 \mathrm{~N}-\mathrm{m}$. Compared to all other motors induction motor is most reliable and robust. The drive performance is also observed at low and medium speed applications with $300 \mathrm{rpm}$ as reference speed. Many techniques are used to model the long feeder. In all those techniques Frequency domain analysis is a powerful tool in feeder modeling [1].Lumped $\mathrm{L}$ and $\mathrm{C}$ elements can represent the feeder effectively, even for transient analysis. Applying this technique is simple and does not relatively scarify the accuracy. Hence this modeling technique is used in this paper. There are many closed-loop techniques used for induction motor speed estimation, in that all techniques model reference adaptive system (MRAS) based on rotor flux [2] is simple, stable. The MRAS speed estimator requires motor voltages and currents as inputs. Direct measurement of the motor voltages and currents is impractical for long feeder drives due to feeder length and safety of environmental precautions. The motor voltages and currents are drawn from the output side of inverter. The proposed model is simulated with 300r.p.m as reference in MATLAB/SIMULINK and observes the outputs speeds with sudden load change of $2 \mathrm{~N}$-m under steady state condition.

This paper is organized into seven sections. Following the introduction in Section 1, MRAS speed estimation is discussed in Section 2. Current source inverter is in Section 3. Analysis of long feeder is discussed in Section 4. Drive system performance is illustrated in Section 5. The simulation of the model is discussed in Section 6. Finally, the conclusion forms Section 7.

\section{Model Reference Adaptive System}

The model reference adaptive system contains two models they are stator model and rotor model. The speed of the machine is estimated by using the above two models [2]. The inputs required for the model reference adaptive system is the voltages and currents. The speed is estimated from the calculation of rotor flux linkages in the stationary reference frame from the both stator and rotor models. The estimated speed is calculated from the error obtained by cross multiplication of the flux linkages produced in the two models (stator and rotor models) with PI controller. The estimated speed is fed back to the one of the input of rotor model. Rotor model is also called as adaptive or adjustable model and the stator model is called the reference model. The two models are designed in Simulink by using the following equations and the block diagram is represented as shown in fig.1.

The equations of reference model as follows 


$$
\begin{aligned}
\psi_{d r}^{s} & =\frac{L_{r}}{L_{m}}\left(\int\left(V_{d s}^{s}-i_{d s}^{s} R_{s}\right) d t-\rho L_{s} i^{s}{ }_{d s}\right) \\
\psi_{q r}^{s} & =\frac{L_{r}}{L_{m}}\left(\int\left(V^{s}{ }_{q s}-i^{s}{ }_{q s} R_{s}\right) d t-\rho L_{s} i^{s}{ }_{q s}\right)
\end{aligned}
$$

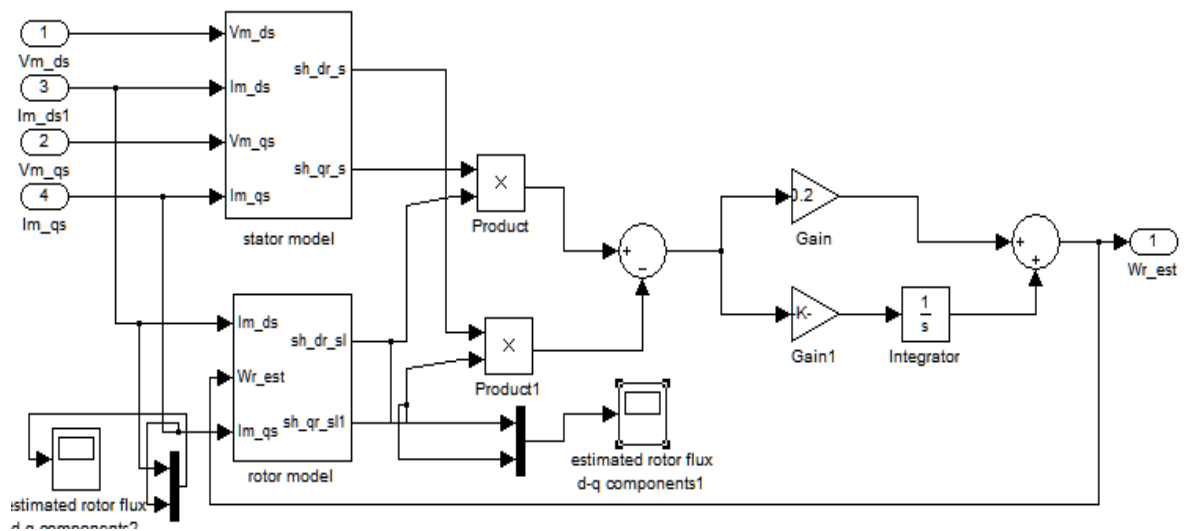

Fig.1Simulation block of MRAS.

The equations of adaptive model is as follows

Error generated from the two models is

$$
\begin{aligned}
\psi_{d r}^{s l} & =\int\left(-\frac{R_{r}}{L_{r}} \psi_{d r}^{s l}-\omega_{r_{-} e s t} \psi_{q r}^{s l}+L_{m} \frac{R_{r}}{L_{r}} i_{d s}^{s}\right) d t \\
\psi_{q r}^{s l} & =\int\left(-\frac{R_{r}}{L_{r}} \psi_{q r}^{s l}+\omega_{r_{-} e s t} \psi_{r}^{s l}+L_{m} \frac{R_{r}}{L_{r}} i_{q s}^{s}\right) d t
\end{aligned}
$$

e_speed $=\left(\psi_{d r}^{s l} \psi_{q r}^{s}-\psi_{q r}^{s l} \psi_{d r}^{s}\right)^{----(5)}$

The estimated aped is calculated as

$$
\omega_{r_{-} e s t}=\left(K_{p}+\frac{K_{i}}{S}\right) e_{-} \text {speed }- \text { - (6) }
$$

The estimated speed is in rad/sec and it can be changed in terms of rpm by multiplying with (30/pi). The estimated speed is considered as the one of the input for indirect vector control.

\section{Current source inverter}

Current Source Inverter was used in many industries from many years to drive the medium and large horsepower motors. Some of the advantages of this converter are its sustained regenerative capabilities, short circuit protection, and it's buffering of the drive output form supply voltage variations. The input to the CSI is obtained by connecting impedance in series with the voltage source. This approach, though it offers excellent performance in steady state.

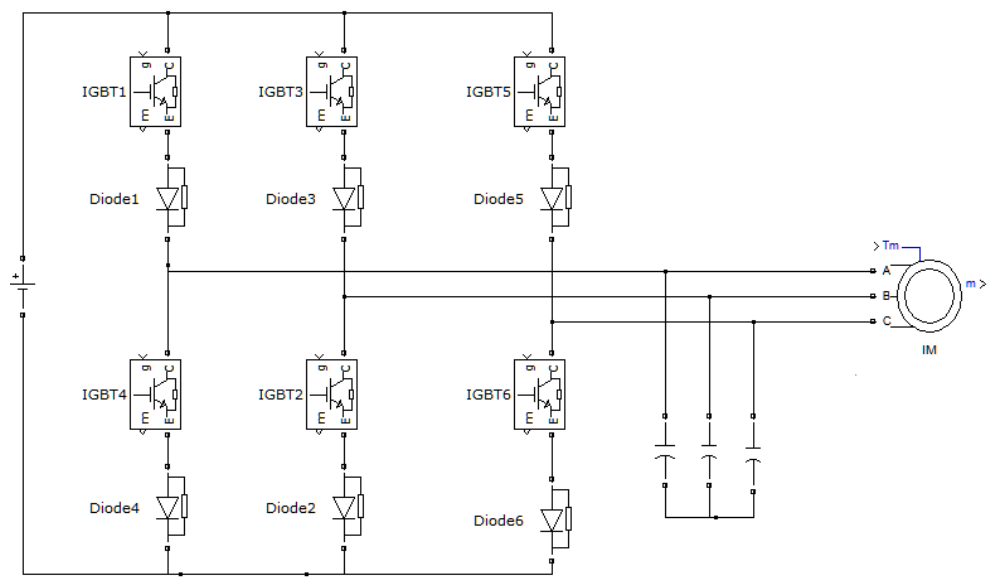

Fig.2. Current source inverter

The above figure shows a high performance CSI; it has a controllable rectifier at the input and an induction machine as a load at the output of the inverter. The current source inverter is operated with pulse width modulation [3]. At each and every instant three devices are operated simultaneously in top and bottom side. Depending on the operation of the devices three phase output voltages and currents are obtained. The PWM CSI requires a three-phase capacitor $C_{f}$ at its output to assist the commutation of the switching devices. 
Otherwise, a high voltage spike would be induced, causing damage to the switching devices. The capacitor also serves as a harmonic filter improving the motor current and voltage waveforms.

\section{Modeling of Feeder}

A $\pi$-network with lumped elements is used to model the long feeder. This model is sufficient to give accurate results at the feeder terminals, even for transient analysis [4]. The simulated feeder is aspecial insulation reinforced three-phase cable with low shunt capacitance.Based on this model, a 1-km prototype for the feeder model is created in MATLAB/SIMULINK using lumped elements. Using the feeder two-port network representation shown in Fig. 3

$\mathrm{Z}_{\mathrm{L}}=\mathrm{R}_{\mathrm{c} \_\mathrm{abc}}+\mathrm{S} \mathrm{L}_{\mathrm{c} \_\mathrm{abc}}$

$\mathrm{Z}_{\mathrm{c}}=\frac{1}{S C_{C_{-} a b c}}$



Fig.3. $\pi$ network Simulation model of Long feeder

\section{Operation of the Drive}

The sensorless operation of CSI fed induction motor drive system uses in this paper utilizes modified PWM reference signals generation. The output voltage and currents of current source inverter are used to operate the model reference adaptive system by converting three phase currents and voltages to d-q component of voltages and currents. The indirect vector controlled method is used to generate the direct and quadrature components. The flux linkages, estimated speed and torque are considered as inputs. The torque signal is generated by giving estimated speed as the input command to the PI controller. The d-q components of currents and speed are produced by indirect vector control. The model of the indirect vector control is represented as shown in fig. 4.

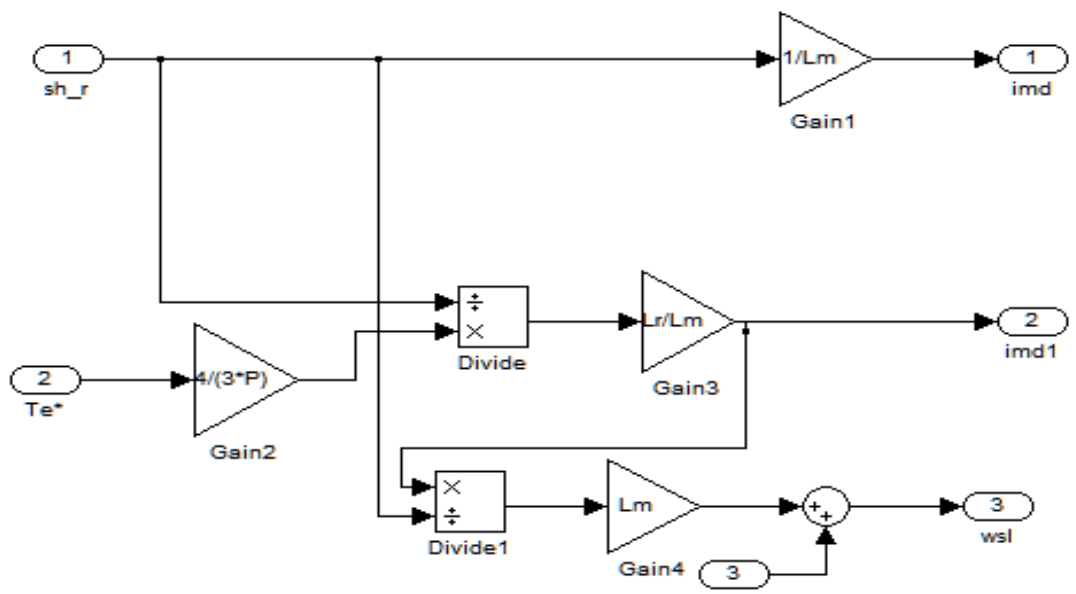

Fig. 4 simulated model of the indirect vector control 
A speed controller to generate torque is

$T^{*}=\left(K_{P}+\frac{K_{I}}{S}\right)\left(N_{e s t}-N_{r}\right)---(9)$

The torque and rotor flux reference commands are utilized to generate the motor $d-q$ reference currents

$$
\begin{aligned}
& i_{m_{-} d s}=\frac{\lambda_{r}}{L_{m}} \\
& i_{m_{-} q s}=\frac{T *}{\left[\frac{3 P L_{m} \lambda_{r}}{4 L_{r}}\right]} \\
& \omega_{s l}=\frac{R_{r}}{L_{r}} \frac{i_{m_{-} q s}}{i_{m_{-} d s}} \\
& \omega_{e}=\omega_{s l}+\omega_{r} \\
& \theta_{e}=\int \omega_{e}
\end{aligned}
$$

The output voltage from the CSI is near sinusoidal, due to the capacitor bank filter action. The CSI is used to control the frequency and phase of the output three-phase currents, the modulating signals to the CSI must have a unity peak. A unit vector generation is needed to generate the modulating signals from the reference $d-q$ currents.

The error produced from the currents of indirect vector control and the component of d-q components is modified to three phase currents; these are used for the production of pulses in the pulse width modulation. The utilization of current PI controllers for Induction Motor (approximately considered as a second order system) CSI-based drives is heavily investigated from the stability point of view under several control techniques(constant $V / f$, direct and indirect vector control, etc).In cases with constant $V / f$ control, the nonoscillating stable region of a PWM system increases since the harmonics caused by the resonance phenomenon are suppressed. Moreover, the negatively damped oscillating operations are caused by the larger bypass capacitors. A capacitor filter design requires deep analysis as it directly affects the drive stability [5]. The motor input is taken from inverter connected through long feeder. The motor is operated and the speed of the motor is analyzed with respect to the estimated speed of the model reference adaptive system.

\section{MATLAB Modeling and Simulation Results}

The proposed model is designed in Simulink to observe the steady state analysis of the current source inverter fed induction motor drive. The simulation model is represented as shown in fig. 5. The CSI sub system consists of current source inverter and the input of the CSI is considered as DC current. Here we have to use the voltage source in series with the high impedance used as a current source for input of current source inverter. The current source inverter is connected to the induction motor drive via long feeder having $1 \mathrm{Km}$ of length. The load varied suddenly by $2 \mathrm{~N}-\mathrm{m}$ at $2 \mathrm{~ms}$. The steady state analysis is observed up to $5 \mathrm{~ms}$.

The Simulated output current wave forms of the current source inverter are shown in fig 6.

The estimated and actual speed of the Model reference adaptive system and the induction motor is shown in fig.7.

The estimated rotor flux $\mathrm{d}-\mathrm{q}$ components and the X-Y mode of estimated rotor flux $\mathrm{d}-\mathrm{q}$ components are shown in fig. 8 and fig. 9 . 


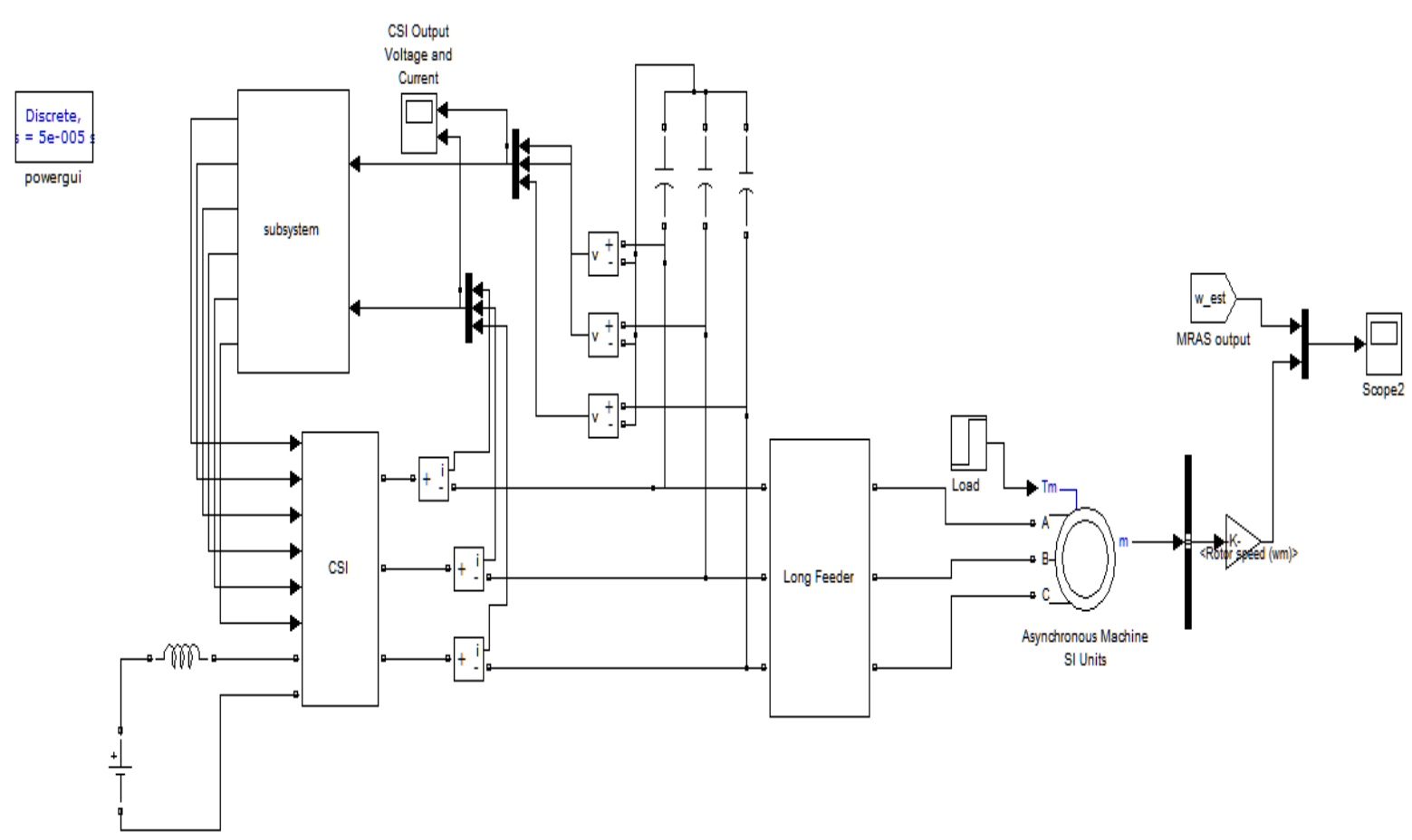

Fig.5. Proposed Model of MRAS based sensorless speed control of CSI fed induction motor drive with long feeder

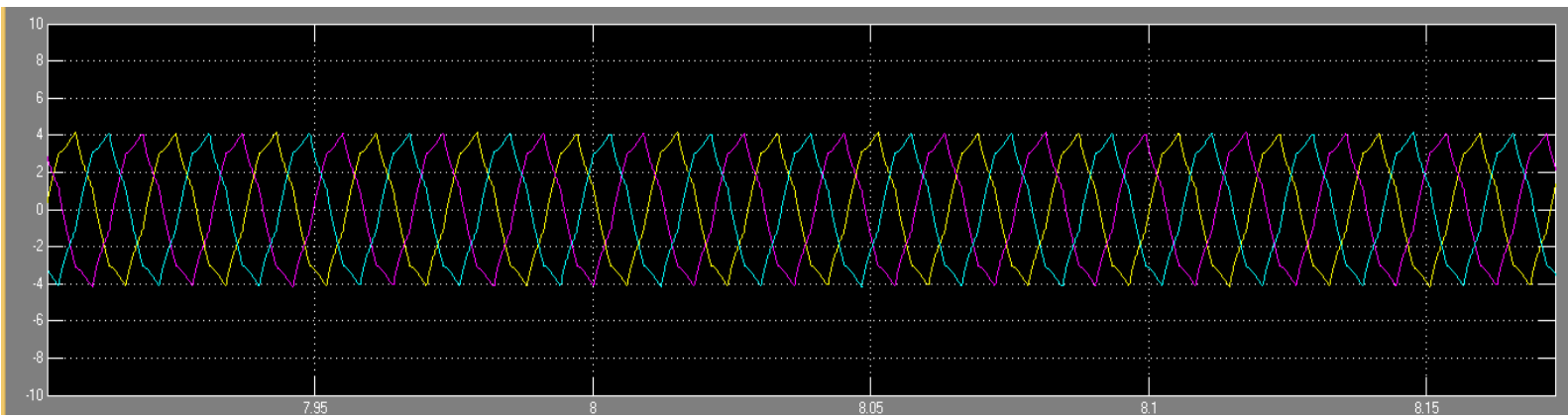

Fig.6. Simulated wave forms of Current source inverter output current waveform



Fig.7. Simulated wave forms of speed estimated by model reference adaptive system and the actual speed of the Induction motor drive. 




Fig.8. Simulated wave forms of estimated rotor flux d-q component.

\section{Conclusion}

The paper is analyzed the MRAS based sensorless speed control of current source fed induction motor drive with long feeder is simulated and the results have shown acceptable performance. The transient and steady state performance of actual and estimated are improved. Finally the proposed technique is moderate and highspeed ranges due to the need of peak detections that requires at least half cycle of the stator frequency.

\section{References}

[1] Ahmed K. Abdelsalam, Mostafa S. Hamad, "Improved Sensorless Operation of a CSI-Based Induction Motor Drive: Long Feeder Case" IEEE trans. Power Electronics., vol. 28 n0. 8, pp. 4001-4012, Aug. 2013.

[2]. C. Schauder, "Adaptive speed identification for vector control of induction motors without rotational transducers," IEEE Trans. Ind. Appl., vol. 28, no. 5, pp. 1054-1061, Sep./Oct. 1992.

[3] K. P. Phillips, "Current-source converter for AC motor drives," IEEE Trans. Ind. Appl., vol. IA-8, no. 6, pp. 679-683, Nov./Dec. 1972.

[4] N. Aoki, K. Satoh, and A. Nabae, "Damping circuit to suppress motor terminal overvoltage and ringing in PWM inverter-fed AC motor drive systems with long motor leads," IEEE Trans. Ind. Appl., vol. 35, no. 5, pp. 1014-1020, Sep./Oct. 1999.

[5] B.Wu, High Power Converter Systems. Piscataway, NJ/NewYork: IEEEPress and Wiley, 2006.

[6] D. Ma, B. Wu, and S. Rizzo, "A CSI-based medium voltage multi-motor drive," in Proc. 3rd Int. Power Electron. Motion Control Conf., 2000, vol. 2, pp. 780-785. 